\title{
Asymptotic behavior of the time-dependent solution of an M/G/1 queueing model
}

Geni Gupur $^{1 *}$ and Rena Ehmet ${ }^{2}$

"Correspondence:

genigupur@yahoo.cn;

geni@xju.edu.cn

${ }^{1}$ College of Mathematics and

Systems Science, Xinjiang

University, Urumai, 830046, P.R.

China

Full list of author information is available at the end of the article

\begin{abstract}
We study the spectrum on the imaginary axis of the underlying operator which corresponds to the $\mathrm{M} / \mathrm{G} / 1$ queueing model with exceptional service time for the first customer in each busy period that was described by infinitely many partial differential equations with integral boundary conditions and obtain that all points on the imaginary axis except 0 belong to the resolvent set of the operator and 0 is an eigenvalue of the operator and its adjoint operator. Thus, by combining these results with our previous results, we deduce that the time-dependent solution of the model converges strongly to its steady-state solution. Moreover, we show that our result on convergence is optimal.
\end{abstract}

MSC: 47A10; 47D99

Keywords: M/G/1 queueing model with exceptional service time for the first customer in each busy period; $C_{0}$-semigroup; eigenvalue; resolvent set

\section{Introduction}

According to Takagi [1], the M/G/1 queueing system with exceptional service time for the first customer in each busy period can be described by the following partial differential equations with integral boundary conditions:

$$
\begin{aligned}
& \frac{\mathrm{d} p_{0}(t)}{\mathrm{d} t}=-\lambda p_{0}(t)+\int_{0}^{\infty} Q_{1}(x, t) b_{0}(x) \mathrm{d} x+\int_{0}^{\infty} p_{1}(x, t) b(x) \mathrm{d} x, \\
& \frac{\partial p_{1}(x, t)}{\partial t}+\frac{\partial p_{1}(x, t)}{\partial x}=-(\lambda+b(x)) p_{1}(x, t), \\
& \frac{\partial p_{k}(x, t)}{\partial t}+\frac{\partial p_{k}(x, t)}{\partial x}=-(\lambda+b(x)) p_{k}(x, t)+\lambda p_{k-1}(x, t), \quad k \geq 2, \\
& \frac{\partial Q_{1}(x, t)}{\partial t}+\frac{\partial Q_{1}(x, t)}{\partial x}=-\left(\lambda+b_{0}(x)\right) Q_{1}(x, t), \\
& \frac{\partial Q_{k}(x, t)}{\partial t}+\frac{\partial Q_{k}(x, t)}{\partial x}=-\left(\lambda+b_{0}(x)\right) Q_{k}(x, t)+\lambda Q_{k-1}(x, t), \quad k \geq 2, \\
& p_{k}(0, t)=\int_{0}^{\infty} Q_{k+1}(x, t) b_{0}(x) \mathrm{d} x+\int_{0}^{\infty} p_{k+1}(x, t) b(x) \mathrm{d} x, \quad k \geq 1, \\
& Q_{1}(0, t)=\lambda p_{0}(t), \quad Q_{k}(0, t)=0, \quad k \geq 2, \\
& p_{0}(0)=1, \quad p_{k}(x, 0)=0, \quad Q_{k}(x, 0)=0, \quad k \geq 1,
\end{aligned}
$$

C 2013 Gupur and Ehmet; licensee Springer. This is an Open Access article distributed under the terms of the Creative Commons Attribution License (http://creativecommons.org/licenses/by/2.0), which permits unrestricted use, distribution, and reproduction in any medium, provided the original work is properly cited. 
where $(x, t) \in[0, \infty) \times[0, \infty) ; p_{0}(t)$ represents the probability that there is no customer in the system and the server is idle at time $t ; p_{n}(x, t) \mathrm{d} x(n \geq 1)$ represents the probability that at time $t$ there are $n$ customers in the system and the server is busy with remaining service time lying between in $[x, x+\mathrm{d} x) ; Q_{n}(x, t) \mathrm{d} x(n \geq 1)$ represents the probability that at time $t$ there are $n$ customers in the system and the server is busy with the elapsed service time of the first service lying between $x$ and $x+\mathrm{d} x ; \lambda$ represents the arrival rate of customers; $b(x)$ is the service rate at $x ; b_{0}(x)$ is the exceptional service rate at $x$.

Many papers have been published about queueing systems with server vacations. But most works on vacation models have been limited to the analysis of steady-states. There are few treatments of transient behavior, see Welch [2], Minh [3], Takagi [1], Gupur [4, 5] for instance. In 1990, Takagi [1] first established the mathematical model of the M/G/1 queueing system with exceptional service time for the first customer in each busy period by using the supplementary variable technique, then studied the time-dependent solution of the model by using probability generating functions and got the Laplace transform of the probability generating function. Roughly speaking, he obtained the existence of a timedependent solution of the model. In 2002, by using $C_{0}$-semigroup theory in functional analysis, Gupur [6] proved that the model has a unique positive time-dependent solution which satisfies the probability condition. In 2003, Gupur [4] considered the asymptotic behavior of the time-dependent solution of the model when $b(x)$ and $b_{0}(x)$ are constants. Firstly, he determined the resolvent set of the adjoint operator of the operator corresponding to the model; next he proved that 0 is an eigenvalue of the operator and its adjoint operator with geometric multiplicity one. Thus, by using Theorem 14 in Gupur, Li and Zhu [7] obtained that the time-dependent solution of the model converges strongly to its steadystate solution. In 2009, Zhang and Gupur [8] found that the operator has one eigenvalue on the left complex half-plane. In 2011, Lin and Gupur [9] proved that the operator has infinitely many eigenvalues on the left complex half-plane which converges to zero and therefore showed that the convergence of the time-dependent solution of the model obtained in Gupur [4] is the best result on the convergence, that is to say, it is impossible that the time-dependent solution exponentially (uniformly) converges to its steady-state solution. In the case that $b(x)$ and $b_{0}(x)$ are functions, any literature about asymptotic behavior of the above model has not been found. This paper is an effort on this subject.

According to Theorem 14 in Gupur, Li and Zhu [7], to obtain the asymptotic behavior of the time-dependent solution of the above model, we need to know the spectrum of the underlying operator on the imaginary axis. By investigating the above model and comparing with Gupur [10], one may find that the main difficult points of the above equations (1.1)-(1.8) are that there are infinitely many equations and boundary conditions. When studying the population equation, Greiner [11] put forward an idea to perturb the boundary condition which states 'one can introduce the maximal operator without the boundary condition and define a boundary operator, and by studying the spectrum of the boundary operator and the maximal operator can discuss the spectrum of the underlying operator which corresponds to the population equation. In 2007, Haji and Radl [12] successfully applied Greiner's idea to the $M / M^{B} / 1$ queueing model, in which both the service rate and arrival rate are constants, and studied the asymptotic behavior of its time-dependent solution. Gupur $[5,13]$ obtained the asymptotic behavior of the time-dependent solutions of two queueing models by using Greiner's idea. In this paper, firstly, by using probability generating functions, we prove that 0 is an eigenvalue of the underlying operator; next, 
by using the idea in Gupur [5, 13], the result in Haji and Radl [12] and Corollary 2.3 in Nagel [14], we deduce the resolvent set of the underlying operator; thirdly, we show that 0 is an eigenvalue of the adjoint operator of the underlying operator, and therefore, by using Theorem 14 in Gupur, Li and Zhu [7], we obtain that the time-dependent solution of the above model converges strongly to its steady-state solution. Finally, by Lin and Gupur [9] we show that our result on convergence is optimal, that is to say, it is impossible that the time-dependent solution of the model converges exponentially to its steady-state solution. Although the idea and method in Gupur [4] are quite different, the main result is a special case of our result.

In this paper, we use the notations in Gupur $[5,6,13]$. Take the state space as follows:

$$
X=\left\{\begin{array}{l|l}
(p, Q) & \begin{array}{l}
p \in \mathbb{R} \times L^{1}[0, \infty) \times L^{1}[0, \infty) \times L^{1}[0, \infty) \times \cdots, \\
Q \in L^{1}[0, \infty) \times L^{1}[0, \infty) \times L^{1}[0, \infty) \times \cdots, \\
\|(p, Q)\|=\left|p_{0}\right|+\sum_{n=1}^{\infty}\left\|p_{n}\right\|_{L^{1}[0, \infty)}+\sum_{n=1}^{\infty}\left\|Q_{n}\right\|_{L^{1}[0, \infty)}<\infty
\end{array}
\end{array}\right\} .
$$

It is obvious that $X$ is a Banach space. In addition, $X$ is also a Banach lattice under the following order relation:

$$
(p, Q) \leq(y, z) \quad \Longleftrightarrow \quad p_{0} \leq y_{0}, \quad p_{n}(x) \leq y_{n}(x), \quad Q_{n}(x) \leq z_{n}(x), \quad n \geq 1 .
$$

For convenience, we introduce

$$
\begin{aligned}
& B_{1} g(x)=-\frac{d g(x)}{d x}-(\lambda+b(x)) g(x), \quad g \in W^{1,1}[0, \infty), \\
& B_{2} g(x)=-\frac{d g(x)}{d x}-\left(\lambda+b_{0}(x)\right) g(x), \quad g \in W^{1,1}[0, \infty), \\
& \phi f(x)=\int_{0}^{\infty} b_{0}(x) f(x) \mathrm{d} x, \quad f \in L^{1}[0, \infty), \\
& \psi f(x)=\int_{0}^{\infty} b(x) f(x) \mathrm{d} x, \quad f \in L^{1}[0, \infty) .
\end{aligned}
$$

We define

$$
\begin{aligned}
A_{m}(p, Q) & =\left(\begin{array}{ccccc}
-\lambda & \psi & 0 & 0 & \ldots \\
0 & B_{1} & 0 & 0 & \ldots \\
0 & \lambda & B_{1} & 0 & \ldots \\
0 & 0 & \lambda & B_{1} & \ldots \\
\vdots & \vdots & \vdots & \vdots & \ddots
\end{array}\right)\left(\begin{array}{c}
p_{0} \\
p_{1}(x) \\
p_{2}(x) \\
p_{3}(x) \\
\vdots
\end{array}\right) \\
& +\left(\begin{array}{ccc}
\phi & 0 & \ldots \\
0 & 0 & \ldots \\
0 & 0 & \ldots \\
\vdots & \vdots & \ddots
\end{array}\right)\left(\begin{array}{c}
Q_{1}(x) \\
Q_{2}(x) \\
Q_{3}(x) \\
\vdots
\end{array}\right)
\end{aligned}
$$




$$
\begin{gathered}
\left.\left(\begin{array}{cccc}
B_{2} & 0 & 0 & \ldots \\
\lambda & B_{2} & 0 & \ldots \\
0 & \lambda & B_{2} & \ldots \\
\vdots & \vdots & \vdots & \ddots
\end{array}\right)\left(\begin{array}{c}
Q_{1}(x) \\
Q_{2}(x) \\
Q_{3}(x) \\
\vdots
\end{array}\right)\right), \\
D\left(A_{m}\right)=\left\{\begin{array}{l|l}
(p, Q) \in X \mid \begin{array}{l}
\frac{\mathrm{d} p_{n}(x)}{\mathrm{d} x} \in L^{1}[0, \infty), \frac{\mathrm{d} Q_{n}(x)}{\mathrm{d} x} \in L^{1}[0, \infty), n \geq 1, \\
p_{n}(x) \text { and } Q_{n}(x) \text { are absolutely continuous functions } \\
\text { and } \sum_{n=1}^{\infty}\left\|\frac{\mathrm{d} p_{n}}{\mathrm{~d} x}\right\|_{L^{1}[0, \infty)}<\infty, \sum_{n=1}^{\infty}\left\|\frac{\mathrm{d} Q_{n}}{\mathrm{~d} x}\right\|_{L^{1}[0, \infty)}<\infty
\end{array}
\end{array}\right\} .
\end{gathered}
$$

We choose a boundary space as

$$
\partial X=l^{1} \times l^{1}
$$

and define the boundary operators

$$
\begin{aligned}
& L: D\left(A_{m}\right) \rightarrow \partial X, \quad \Phi: D\left(A_{m}\right) \rightarrow \partial X \\
& L(p, Q)=L\left(\left(\begin{array}{c}
p_{0} \\
p_{1}(x) \\
p_{2}(x) \\
\vdots
\end{array}\right),\left(\begin{array}{c}
Q_{1}(x) \\
Q_{2}(x) \\
Q_{3}(x) \\
\vdots
\end{array}\right)\right)=\left(\left(\begin{array}{c}
p_{1}(0) \\
p_{2}(0) \\
p_{3}(0) \\
\vdots
\end{array}\right),\left(\begin{array}{c}
Q_{1}(0) \\
Q_{2}(0) \\
Q_{3}(0) \\
\vdots
\end{array}\right)\right), \\
& \Phi(p, Q)=\left(\begin{array}{cccccc}
0 & 0 & \psi & 0 & 0 & \ldots \\
0 & 0 & 0 & \psi & 0 & \ldots \\
0 & 0 & 0 & 0 & \psi & \ldots \\
\vdots & \vdots & \vdots & \vdots & \vdots & \ddots
\end{array}\right)\left(\begin{array}{c}
p_{0} \\
p_{1}(x) \\
p_{2}(x) \\
\vdots
\end{array}\right)+\left(\begin{array}{ccccc}
0 & \phi & 0 & 0 & \ldots \\
0 & 0 & \phi & 0 & \ldots \\
0 & 0 & 0 & \phi & \ldots \\
\vdots & \vdots & \vdots & \vdots & \ddots
\end{array}\right)\left(\begin{array}{c}
Q_{1}(x) \\
Q_{2}(x) \\
Q_{3}(x) \\
\vdots
\end{array}\right), \\
&\left.\left(\begin{array}{cccc}
\lambda & 0 & 0 & \ldots \\
0 & 0 & 0 & \ldots \\
0 & 0 & 0 & \ldots \\
\vdots & \vdots & \vdots & \vdots
\end{array}\right)\left(\begin{array}{c}
p_{0} \\
p_{1}(x) \\
p_{2}(x) \\
\vdots
\end{array}\right)\right) .
\end{aligned}
$$

Now we introduce the underlying operator $(A, D(A))$ by

$$
A p=A_{m} p, \quad D(A)=\left\{p \in D\left(A_{m}\right) \mid L p=\Phi p\right\} .
$$

Then the system of the above equations (1.1)-(1.8) can be written as an abstract Cauchy problem in the Banach space $X$, which is just the form given in Gupur [6]

$$
\left\{\begin{array}{l}
\frac{\mathrm{d}(p, Q)(t)}{\mathrm{d} t}=A(p, Q)(t), \quad t \in(0, \infty), \\
(p, Q)(0)=\left(\left(\begin{array}{l}
1 \\
0 \\
\vdots
\end{array}\right),\left(\begin{array}{l}
0 \\
0 \\
\vdots
\end{array}\right)\right)
\end{array}\right.
$$

Gupur [6] has proved the following result for the system (1.9).

Theorem 1.1 The operator $(A, D(A))$ generates a positive contraction $C_{0}$-semigroup $T(t)$ and the system (1.9) has a unique positive time-dependent solution $(p, Q)(x, t)=$ 
$T(t)(p, Q)(0)$ which satisfies

$$
\begin{aligned}
\|(p, Q)(\cdot, t)\|= & p_{0}(t)+\sum_{n=1}^{\infty} \int_{0}^{\infty} p_{n}(x, t) \mathrm{d} x \\
& +\sum_{n=1}^{\infty} \int_{0}^{\infty} Q_{n}(x, t) \mathrm{d} x=1, \quad \forall t \in[0, \infty) .
\end{aligned}
$$

\section{Main results}

Lemma 2.1 If $\int_{0}^{\infty} \lambda x b(x) e^{-\int_{0}^{x} b(\xi) d \xi} d x<1$, then 0 is an eigenvalue of $A$ with geometric multiplicity one.

Proof We consider the equation $A(p, Q)=0$, i.e.,

$$
\begin{aligned}
& \lambda p_{0}=\int_{0}^{\infty} Q_{1}(x) b_{0}(x) \mathrm{d} x+\int_{0}^{\infty} p_{1}(x) b(x) \mathrm{d} x, \\
& \frac{\mathrm{d} p_{1}(x)}{\mathrm{d} x}=-(\lambda+b(x)) p_{1}(x), \\
& \frac{\mathrm{d} p_{n}(x)}{\mathrm{d} x}=-(\lambda+b(x)) p_{n}(x)+\lambda p_{n-1}(x), \quad \forall n \geq 2, \\
& \frac{\mathrm{d} Q_{1}(x)}{\mathrm{d} x}=-\left(\lambda+b_{0}(x)\right) Q_{1}(x), \\
& \frac{\mathrm{d} Q_{n}(x)}{\mathrm{d} x}=-\left(\lambda+b_{0}(x)\right) Q_{n}(x)+\lambda Q_{n-1}(x), \quad \forall n \geq 2, \\
& p_{n}(0)=\int_{0}^{\infty} Q_{n+1}(x) b_{0}(x) \mathrm{d} x+\int_{0}^{\infty} p_{n+1}(x) b(x) \mathrm{d} x, \quad n \geq 1, \\
& Q_{1}(0)=\lambda p_{0}, \quad Q_{k}(0)=0, \quad k \geq 2 .
\end{aligned}
$$

By solving (2.2)-(2.5), we have

$$
\begin{aligned}
p_{1}(x)= & a_{1} e^{-\lambda x-\int_{0}^{x} b(\xi) \mathrm{d} \xi}, \\
p_{n}(x)= & a_{n} e^{-\lambda x-\int_{0}^{x} b(\xi) \mathrm{d} \xi} \\
& +\lambda e^{-\lambda x-\int_{0}^{x} b(\xi) \mathrm{d} \xi} \int_{0}^{x} p_{n-1}(s) e^{\lambda s+\int_{0}^{s} b(\xi) \mathrm{d} \xi} \mathrm{d} s, \quad n \geq 2, \\
Q_{1}(x)= & b_{1} e^{-\lambda x-\int_{0}^{x} b_{0}(\xi) \mathrm{d} \xi}, \\
Q_{n}(x)= & b_{n} e^{-\lambda x-\int_{0}^{x} b_{0}(\xi) \mathrm{d} \xi} \\
& +\lambda e^{-\lambda x-\int_{0}^{x} b_{0}(\xi) \mathrm{d} \xi} \int_{0}^{x} Q_{n-1}(s) e^{\lambda s+\int_{0}^{s} b_{0}(\xi) \mathrm{d} \xi} \mathrm{d} s, \quad n \geq 2 .
\end{aligned}
$$

Through using (2.8)-(2.11) repeatedly, we deduce

$$
\begin{aligned}
& p_{n}(x)=e^{-\lambda x-\int_{0}^{x} b(\xi) \mathrm{d} \xi} \sum_{k=1}^{n} \frac{(\lambda x)^{k-1}}{(k-1) !} a_{n+1-k}, \quad n \geq 1, \\
& Q_{n}(x)=e^{-\lambda x-\int_{0}^{x} b_{0}(\xi) \mathrm{d} \xi} \sum_{k=1}^{n} \frac{(\lambda x)^{k-1}}{(k-1) !} b_{n+1-k}, \quad n \geq 1 .
\end{aligned}
$$


By combining (2.10) and (2.11) with (2.7) and using (2.13), we deduce

$$
\begin{aligned}
Q_{n}(x) & =\lambda p_{0} \frac{(\lambda x)^{n-1}}{(n-1) !} e^{-\lambda x-\int_{0}^{x} b_{0}(\xi) d \xi}, \quad n \geq 1 \\
\Longrightarrow & \\
\sum_{n=1}^{\infty}\left\|Q_{n}\right\|_{L^{1}[0, \infty)} & =\sum_{n=1}^{\infty} \int_{0}^{\infty}\left|\lambda p_{0} \frac{(\lambda x)^{n-1}}{(n-1) !}\right| e^{-\lambda x-\int_{0}^{x} b_{0}(\xi) d \xi} d x \\
& =\lambda\left|p_{0}\right| \int_{0}^{\infty} \sum_{n=1}^{\infty} \frac{(\lambda x)^{n-1}}{(n-1) !} e^{-\lambda x-\int_{0}^{x} b_{0}(\xi) d \xi} d x \\
& =\lambda\left|p_{0}\right| \int_{0}^{\infty} e^{\lambda x} e^{-\lambda x-\int_{0}^{x} b_{0}(\xi) d \xi} d x \\
& =\lambda\left|p_{0}\right| \int_{0}^{\infty} e^{-\int_{0}^{x} b_{0}(\xi) d \xi} d \xi<\infty .
\end{aligned}
$$

It is difficult to determine directly all $a_{k}$ and to verify $\sum_{k=1}^{\infty}\left\|p_{k}\right\|_{L^{1}[0, \infty)}<\infty$. In the following, we use another method. We introduce the probability generating function $P(x, z)=$ $\sum_{n=1}^{\infty} p_{n}(x) z^{n}$ for all complex variables $|z|<1$. Theorem 1.1 ensures that $P(x, z)$ is well defined. (2.2) and (2.3) give

$$
\begin{aligned}
& \frac{\partial \sum_{n=1}^{\infty} p_{n}(x) z^{n}}{\partial x}=-\sum_{n=1}^{\infty}(\lambda+b(x)) p_{n}(x) z^{n}+\lambda \sum_{n=2}^{\infty} p_{n-1}(x) z^{n}, \\
& \frac{\partial P(x, z)}{\partial x}=-(\lambda+b(x)) \sum_{n=1}^{\infty} p_{n}(x) z^{n}+\lambda z \sum_{n=1}^{\infty} p_{n}(x) z^{n}, \\
& \frac{\partial P(x, z)}{\partial x}=-(\lambda+b(x)) P(x, z)+\lambda z P(x, z)=[\lambda(z-1)-b(x)] P(x, z) \\
& \Longrightarrow \\
& P(x, z)=P(0, z) e^{\lambda x(z-1)-\int_{0}^{x} b(\xi) \mathrm{d} \xi} .
\end{aligned}
$$

By applying (2.6), (2.16), (2.14), (2.1), $\int_{0}^{\infty} b(x) e^{-\int_{0}^{x} b(\xi) d \xi} d x=1, \int_{0}^{\infty} b_{0}(x) e^{-\int_{0}^{x} b_{0}(\xi) d \xi} d x=1$ and the L'Hospital rule it follows that

$$
\begin{aligned}
P(0, z)= & \sum_{n=1}^{\infty} p_{n}(0) z^{n}=\sum_{n=1}^{\infty}\left(\int_{0}^{\infty} Q_{n+1}(x) b_{0}(x) \mathrm{d} x+\int_{0}^{\infty} p_{n+1}(x) b(x) \mathrm{d} x\right) z^{n} \\
= & \int_{0}^{\infty} b_{0}(x) \sum_{n=1}^{\infty} Q_{n+1}(x) z^{n} d x+\int_{0}^{\infty} b(x) \sum_{n=1}^{\infty} p_{n+1}(x) z^{n} d x \\
= & \int_{0}^{\infty} b_{0}(x) \sum_{n=1}^{\infty} \lambda p_{0} \frac{(\lambda x)^{n}}{n !} z^{n} e^{-\lambda x-\int_{0}^{x} b_{0}(\xi) d \xi} d x \\
& +\int_{0}^{\infty} b(x) \frac{1}{z} \sum_{n=1}^{\infty} p_{n+1}(x) z^{n+1} d x \\
= & \lambda p_{0} \int_{0}^{\infty} b_{0}(x) e^{\lambda x z} e^{-\lambda x-\int_{0}^{x} b_{0}(\xi) d \xi} d x
\end{aligned}
$$




$$
\begin{aligned}
& +\int_{0}^{\infty} b(x) \frac{1}{z}\left\{\sum_{n=1}^{\infty} p_{n}(x) z^{n}-p_{1}(x) z\right\} d x \\
= & \lambda p_{0} \int_{0}^{\infty} b_{0}(x) e^{\lambda x(z-1)-\int_{0}^{x} b_{0}(\xi) d \xi} d x \\
& +\frac{1}{z} \int_{0}^{\infty} b(x) \sum_{n=1}^{\infty} p_{n}(x) z^{n} d z-\int_{0}^{\infty} b(x) p_{1}(x) d x \\
= & \lambda p_{0} \int_{0}^{\infty} b_{0}(x) e^{\lambda x(z-1)-\int_{0}^{x} b_{0}(\xi) d \xi} d x \\
& +\frac{1}{z} \int_{0}^{\infty} b(x) \sum_{n=1}^{\infty} p_{n}(x) z^{n} d z-\left[\lambda p_{0}-\int_{0}^{\infty} \lambda p_{0} b_{0}(x) e^{-\lambda x-\int_{0}^{x} b_{0}(\xi) d \xi} d x\right] \\
= & \lambda p_{0} \int_{0}^{\infty} b_{0}(x) e^{\lambda x(z-1)-\int_{0}^{x} b_{0}(\xi) d \xi} d x-\lambda p_{0} \\
& +\lambda p_{0} \int_{0}^{\infty} b_{0}(x) e^{-\lambda x-\int_{0}^{x} b_{0}(\xi) d \xi} d x+\frac{1}{z} \int_{0}^{\infty} b(x) P(0, z) e^{\lambda x(z-1)-\int_{0}^{x} b(\xi) d \xi} d x \\
\Longrightarrow & \\
P(0, z)= & \frac{\int_{0}^{\infty} b_{0}(x) e^{\lambda x(z-1)-\int_{0}^{x} b_{0}(\xi) d \xi} d x-1+\int_{0}^{\infty} b_{0}(x) e^{-\lambda x-\int_{0}^{x} b_{0}(\xi) d \xi} d x}{z-\int_{0}^{\infty} b(x) e^{\lambda x(z-1)-\int_{0}^{x} b(\xi) \mathrm{d} \xi} \mathrm{d} x} \\
\Longrightarrow & \Rightarrow p_{0} z \\
\lim _{z \rightarrow 1} P(0, z)= & \frac{\int_{0}^{\infty} \lambda x b_{0}(x) e^{-\int_{0}^{x} b_{0}(\xi) d \xi} d x+\int_{0}^{\infty} b_{0}(x) e^{-\lambda x-\int_{0}^{x} b_{0}(\xi) d \xi} d x}{1-\int_{0}^{\infty} \lambda x b(x) e^{-\int_{0}^{x} b(\xi) d \xi} d x} .
\end{aligned}
$$

(2.16) and (2.17) give

$$
\begin{aligned}
\sum_{n=1}^{\infty} p_{n}(x)= & \lim _{z \rightarrow 1} P(x, z) \\
= & \frac{\int_{0}^{\infty} \lambda x b_{0}(x) e^{-\int_{0}^{x} b_{0}(\xi) d \xi} d x+\int_{0}^{\infty} b_{0}(x) e^{-\lambda x-\int_{0}^{x} b_{0}(\xi) d \xi} d x}{1-\int_{0}^{\infty} \lambda x b(x) e^{-\int_{0}^{x} b(\xi) d \xi} d x} \lambda\left|p_{0}\right| \\
& \times \int_{0}^{\infty} e^{-\int_{0}^{x} b(\xi) d \xi} d x \\
< & \infty
\end{aligned}
$$

(2.18) and (2.15) show that 0 is an eigenvalue of $A$. Moreover, from (2.12), (2.14), (2.1) and (2.6), it is easy to see that the eigenvectors corresponding to zero span one dimensional linear space, that is, the geometric multiplicity of 0 is one.

According to Theorem 14 in Gupur, Li and Zhu [7], we know that in order to obtain the asymptotic behavior of the time-dependent solution of the system (1.9), we need the spectrum of $A$ on the imaginary axis. Through investigating the system (1.9), we find that the infinite number of equations and the boundary conditions are the difficult points. Greiner [11] put forward an idea to study the spectrum of $A$ by perturbing boundary conditions. And by using the Greiner idea, Haji and Radl [12] gave a result which was described by the Dirichlet operator. In the following, by applying the result, we deduce the resolvent set of 
$A$ on the imaginary axis. To do this, define $\left(A_{0}, D\left(A_{0}\right)\right)$ as

$$
A_{0} p=A_{m} p, \quad D\left(A_{0}\right)=\left\{p \in D\left(A_{m}\right) \mid L p=0\right\}
$$

and discuss the inverse of $A_{0}$. For any given $(y, z) \in X$, consider the equation $(\gamma I-$ $\left.A_{0}\right)(p, Q)=(y, z)$, that is,

$$
\begin{aligned}
& (\gamma+\lambda) p_{0}=y_{0}+\int_{0}^{\infty} Q_{1}(x) b_{0}(x) \mathrm{d} x+\int_{0}^{\infty} p_{1}(x) b(x) \mathrm{d} x, \\
& \frac{\mathrm{d} p_{1}(x)}{\mathrm{d} x}=-(\gamma+\lambda+b(x)) p_{1}(x)+y_{1}(x), \\
& \frac{\mathrm{d} p_{n}(x)}{\mathrm{d} x}=-(\gamma+\lambda+b(x)) p_{n}(x)+\lambda p_{n-1}(x)+y_{n}(x), \quad \forall n \geq 2, \\
& \frac{\mathrm{d} Q_{1}(x)}{\mathrm{d} x}=-\left(\gamma+\lambda+b_{0}(x)\right) Q_{1}(x)+z_{1}(x), \\
& \frac{\mathrm{d} Q_{n}(x)}{\mathrm{d} x}=-\left(\gamma+\lambda+b_{0}(x)\right) Q_{n}(x)+\lambda Q_{n-1}(x)+z_{n}(x), \quad \forall n \geq 2, \\
& p_{n}(0)=0, \quad Q_{n}(0)=0, \quad n \geq 1 .
\end{aligned}
$$

By (2.19)-(2.24) it is easy to calculate

$$
\begin{aligned}
p_{0}= & \frac{y_{0}}{\gamma+\lambda}+\frac{1}{\gamma+\lambda} \int_{0}^{\infty} b(x) e^{-(\gamma+\lambda) x-\int_{0}^{x} b(\xi) \mathrm{d} \xi} \int_{0}^{x} y_{1}(s) e^{(\gamma+\lambda) s+\int_{0}^{s} b(\xi) \mathrm{d} \xi} \mathrm{d} s \mathrm{~d} x \\
& +\frac{1}{\gamma+\lambda} \int_{0}^{\infty} b_{0}(x) e^{-(\gamma+\lambda) x-\int_{0}^{x} b_{0}(\xi) \mathrm{d} \xi} \int_{0}^{x} z_{1}(s) e^{(\gamma+\lambda) s+\int_{0}^{s} b_{0}(\xi) \mathrm{d} \xi} \mathrm{d} s \mathrm{~d} x, \\
p_{1}(x)= & e^{-(\gamma+\lambda) x-\int_{0}^{x} b(\xi) \mathrm{d} \xi} \int_{0}^{x} y_{1}(s) e^{(\gamma+\lambda) s+\int_{0}^{s} b(\xi) \mathrm{d} \xi} \mathrm{d} s, \\
p_{n}(x)= & e^{-(\gamma+\lambda) x-\int_{0}^{x} b(\xi) \mathrm{d} \xi} \int_{0}^{x} y_{n}(s) e^{(\gamma+\lambda) s+\int_{0}^{s} b(\xi) \mathrm{d} \xi} \mathrm{d} s \\
& +\lambda e^{-(\gamma+\lambda) x-\int_{0}^{x} b(\xi) \mathrm{d} \xi} \int_{0}^{x} p_{n-1}(s) e^{(\gamma+\lambda) s+\int_{0}^{s} b(\xi) \mathrm{d} \xi} \mathrm{d} s, \quad n \geq 2, \\
Q_{1}(x)= & e^{-(\gamma+\lambda) x-\int_{0}^{x} b_{0}(\xi) \mathrm{d} \xi} \int_{0}^{x} z_{1}(s) e^{(\gamma+\lambda) s+\int_{0}^{s} b_{0}(\xi) \mathrm{d} \xi} \mathrm{d} s, \\
Q_{n}(x)= & e^{-(\gamma+\lambda) x-\int_{0}^{x} b_{0}(\xi) \mathrm{d} \xi} \int_{0}^{x} z_{n}(s) e^{(\gamma+\lambda) s+\int_{0}^{s} b_{0}(\xi) \mathrm{d} \xi} \mathrm{d} s \\
& +\lambda e^{-(\gamma+\lambda) x-\int_{0}^{x} b_{0}(\xi) \mathrm{d} \xi} \int_{0}^{x} Q_{n-1}(s) e^{(\gamma+\lambda) s+\int_{0}^{s} b_{0}(\xi) \mathrm{d} \xi} \mathrm{d} s, \quad n \geq 2 .
\end{aligned}
$$

If we set

$$
\begin{aligned}
& E f(x)=e^{-(\gamma+\lambda) x-\int_{0}^{x} b(\xi) \mathrm{d} \xi} \int_{0}^{x} f(s) e^{(\gamma+\lambda) s+\int_{0}^{s} b(\xi) \mathrm{d} \xi} \mathrm{d} s, \quad \forall f \in L^{1}[0, \infty), \\
& E_{0} f(x)=e^{-(\gamma+\lambda) x-\int_{0}^{x} b_{0}(\xi) \mathrm{d} \xi} \int_{0}^{x} f(s) e^{(\gamma+\lambda) s+\int_{0}^{s} b_{0}(\xi) \mathrm{d} \xi} \mathrm{d} s, \quad \forall f \in L^{1}[0, \infty),
\end{aligned}
$$


then the above equations (2.25)-(2.29) give, if the resolvent of $A_{0}$ exists,

$$
\begin{aligned}
\left(\gamma I-A_{0}\right)^{-1}(y, z)= & \left(\begin{array}{ccccc}
\frac{1}{\gamma+\lambda} & \frac{1}{\gamma+\lambda} \psi E & 0 & 0 & \ldots \\
0 & E & 0 & 0 & \ldots \\
0 & \lambda E^{2} & E & 0 & \ldots \\
0 & \lambda^{2} E^{3} & \lambda E^{2} & E & \ldots \\
\vdots & \vdots & \vdots & \vdots & \ddots
\end{array}\right)\left(\begin{array}{c}
y_{0} \\
y_{1}(x) \\
y_{2}(x) \\
y_{3}(x) \\
\vdots
\end{array}\right) \\
& +\left(\begin{array}{cccc}
\frac{1}{\gamma+\lambda} \phi E_{0} & 0 & 0 & \ldots \\
0 & 0 & 0 & \ldots \\
0 & 0 & 0 & \ldots \\
\vdots & \vdots & \vdots & \ddots
\end{array}\right)\left(\begin{array}{c}
z_{1}(x) \\
z_{2}(x) \\
z_{3}(x) \\
\vdots
\end{array}\right) \\
& \left.\left(\begin{array}{cccc}
E_{0} & 0 & 0 & \ldots \\
\lambda E_{0}^{2} & E_{0} & 0 & \ldots \\
\lambda^{2} E_{0}^{3} & \lambda E_{0}^{2} & E_{0} & \ldots \\
\vdots & \vdots & \vdots & \ddots
\end{array}\right)\left(\begin{array}{c}
z_{1}(x) \\
z_{2}(x) \\
z_{3}(x) \\
\vdots
\end{array}\right)\right)
\end{aligned}
$$

From which together with the definition of the resolvent set we have the following result.

Lemma 2.2 Let $b(x), b_{0}(x):[0, \infty) \rightarrow[0, \infty)$ be measurable, $0<\inf _{x \in[0, \infty)} b(x) \leq$ $\sup _{x \in[0, \infty)} b(x)<\infty$ and $0<\inf _{x \in[0, \infty)} b_{0}(x) \leq \sup _{x \in[0, \infty)} b_{0}(x)<\infty$. Then

$$
\left\{\begin{array}{l|l}
\gamma \in \mathbb{C} & \begin{array}{l}
\operatorname{Re} \gamma+\lambda>0, \\
\operatorname{Re} \gamma+\inf _{x \in[0, \infty)} b(x)>0, \\
\operatorname{Re} \gamma+\inf _{x \in[0, \infty)} b_{0}(x)>0
\end{array}
\end{array}\right\} \subset \rho\left(A_{0}\right) .
$$

Proof For any $f \in L^{1}[0, \infty)$, by using integration by parts, we estimate

$$
\begin{aligned}
\int_{0}^{\infty}|E f(x)| \mathrm{d} x= & \int_{0}^{\infty}\left|e^{-(\gamma+\lambda) x-\int_{0}^{x} b(\xi) \mathrm{d} \xi} \int_{0}^{x} e^{(\gamma+\lambda) s+\int_{0}^{s} b(\xi) \mathrm{d} \xi} f(s) \mathrm{d} s\right| \mathrm{d} x \\
\leq & \int_{0}^{\infty} e^{-(\operatorname{Re} \gamma+\lambda) x-\int_{0}^{x} b(\xi) \mathrm{d} \xi} \int_{0}^{x} e^{(\operatorname{Re} \gamma+\lambda) s+\int_{0}^{s} b(\xi) \mathrm{d} \xi}|f(s)| \mathrm{d} s \mathrm{~d} x \\
\leq & \frac{-1}{\operatorname{Re} \gamma+\lambda+\inf _{x \in[0, \infty)} b(x)} \\
& \times \int_{0}^{\infty} \int_{0}^{x} e^{(\operatorname{Re} \gamma+\lambda) s+\int_{0}^{s} b(\xi) d \xi}|f(s)| d s d e^{-(\operatorname{Re} \gamma+\lambda) x-\int_{0}^{x} b(\xi) d \xi} \\
= & \frac{-1}{\operatorname{Re} \gamma+\lambda+\inf _{x \in[0, \infty)} b(x)} \\
& \times\left[\left.e^{-(\operatorname{Re} \gamma+\lambda) x-\int_{0}^{x} b(\xi) \mathrm{d} \xi} \int_{0}^{x} e^{(\operatorname{Re} \gamma+\lambda) s+\int_{0}^{s} b(\xi) \mathrm{d} \xi}|f(s)| \mathrm{d} s\right|_{x=0} ^{x=\infty}\right. \\
& \left.-\int_{0}^{\infty} e^{-(\operatorname{Re} \gamma+\lambda) x-\int_{0}^{x} b(\xi) \mathrm{d} \xi} e^{(\operatorname{Re} \gamma+\lambda) x+\int_{0}^{x} b(\xi) \mathrm{d} \xi}|f(x)| \mathrm{d} x\right] \\
= & \frac{1}{\operatorname{Re} \gamma+\lambda+\inf _{x \in[0, \infty)} b(x)} \mid f \|_{L^{1}[0, \infty)}
\end{aligned}
$$




$$
\|E\| \leq \frac{1}{\operatorname{Re} \gamma+\lambda+\inf _{x \in[0, \infty)} b(x)} .
$$

Similarly,

$$
\left\|E_{0}\right\| \leq \frac{1}{\operatorname{Re} \gamma+\lambda+\inf _{x \in[0, \infty)} b_{0}(x)} .
$$

From (2.30), (2.31), $\|\phi\| \leq \sup _{x \in[0, \infty)} b_{0}(x)$ and $\|\psi\| \leq \sup _{x \in[0, \infty)} b(x)$ we deduce, for $(y, z) \in X$,

$$
\begin{aligned}
& \left\|\left(\gamma I-A_{0}\right)^{-1}(y, z)\right\| \\
& =\left|\frac{y_{0}}{\gamma+\lambda}+\frac{1}{\gamma+\lambda} \psi E y_{1}+\frac{1}{\gamma+\lambda} \phi E_{0} z_{1}\right| \\
& +\left\|E y_{1}\right\|_{L^{1}[0, \infty)}+\left\|\lambda E^{2} y_{1}+E y_{2}\right\|_{L^{1}[0, \infty)}+\left\|\lambda^{2} E^{3} y_{1}+\lambda E^{2} y_{2}+E y_{3}\right\|_{L^{1}[0, \infty)} \\
& +\left\|\lambda^{3} E^{4} y_{1}+\lambda^{2} E^{3} y_{2}+\lambda E^{2} y_{3}+E y_{4}\right\|_{L^{1}[0, \infty)} \\
& +\cdots \\
& +\left\|E_{0} z_{1}\right\|_{L^{1}[0, \infty)}+\left\|\lambda E_{0}^{2} z_{1}+E_{0} z_{2}\right\|_{L^{1}[0, \infty)}+\left\|\lambda^{2} E_{0}^{3} z_{1}+\lambda E_{0}^{2} z_{2}+E_{0} z_{3}\right\|_{L^{1}[0, \infty)} \\
& +\cdots \\
& \leq \frac{1}{|\gamma+\lambda|}\left|y_{0}\right|+\frac{1}{|\gamma+\lambda|}\left|\psi E y_{1}\right|+\frac{1}{|\gamma+\lambda|}\left|\phi E_{0} z_{1}\right| \\
& +\left\|E y_{1}\right\|_{L^{1}[0, \infty)}+\left\|\lambda E^{2} y_{1}\right\|_{L^{1}[0, \infty)}+\left\|E y_{2}\right\|_{L^{1}[0, \infty)}+\left\|\lambda^{2} E^{3} y_{1}\right\|_{L^{1}[0, \infty)} \\
& +\left\|\lambda E^{2} y_{2}\right\|_{L^{1}[0, \infty)}+\left\|E y_{3}\right\|_{L^{1}[0, \infty)}+\left\|\lambda^{3} E^{4} y_{1}\right\|_{L^{1}[0, \infty)} \\
& +\left\|\lambda^{2} E^{3} y_{2}\right\|_{L^{1}[0, \infty)}+\left\|\lambda E^{2} y_{3}\right\|_{L^{1}[0, \infty)}+\left\|E y_{4}\right\|_{L^{1}[0, \infty)} \\
& +\cdots \\
& +\left\|E_{0} z_{1}\right\|_{L^{1}[0, \infty)}+\left\|\lambda E_{0}^{2} z_{1}\right\|_{L^{1}[0, \infty)}+\left\|E_{0} z_{2}\right\|_{L^{1}[0, \infty)}+\left\|\lambda^{2} E_{0}^{3} z_{1}\right\|_{L^{1}[0, \infty)} \\
& +\left\|\lambda E_{0}^{2} z_{2}\right\|_{L^{1}[0, \infty)}+\left\|E_{0} z_{3}\right\|_{L^{1}[0, \infty)} \\
& +\cdots \\
& \leq \frac{1}{|\gamma+\lambda|}\left|y_{0}\right|+\frac{1}{|\gamma+\lambda|}\|\psi\|\|E\|\left\|y_{1}\right\|_{L^{1}[0, \infty)}+\frac{1}{|\gamma+\lambda|}\|\phi\|\left\|E_{0}\right\|\left\|z_{1}\right\|_{L^{1}[0, \infty)} \\
& +\|E\|\left\|y_{1}\right\|_{L^{1}[0, \infty)}+\lambda\|E\|^{2}\left\|y_{1}\right\|_{L^{1}[0, \infty)}+\|E\|\left\|y_{2}\right\|_{L^{1}[0, \infty)} \\
& +\lambda^{2}\|E\|^{3}\left\|y_{1}\right\|_{L^{1}[0, \infty)}+\lambda\|E\|^{2}\left\|y_{2}\right\|_{L^{1}[0, \infty)}+\left\|E y_{3}\right\|_{L^{1}[0, \infty)} \\
& +\lambda^{3}\|E\|^{4}\left\|y_{1}\right\|_{L^{1}[0, \infty)}+\lambda^{2}\|E\|^{3}\left\|y_{2}\right\|_{L^{1}[0, \infty)}+\lambda\|E\|^{2}\left\|y_{3}\right\|_{L^{1}[0, \infty)}+\|E\|\left\|y_{4}\right\|_{L^{1}[0, \infty)} \\
& +\cdots \\
& +\left\|E_{0}\right\|\left\|z_{1}\right\|_{L^{1}[0, \infty)}+\lambda\left\|E_{0}\right\|^{2}\left\|z_{1}\right\|_{L^{1}[0, \infty)}+\left\|E_{0}\right\|\left\|z_{2}\right\|_{L^{1}[0, \infty)} \\
& +\lambda^{2}\left\|E_{0}\right\|^{3}\left\|z_{1}\right\|_{L^{1}[0, \infty)}+\lambda\left\|E_{0}\right\|^{2}\left\|z_{2}\right\|_{L^{1}[0, \infty)}+\left\|E_{0}\right\|\left\|z_{3}\right\|_{L^{1}[0, \infty)} \\
& +\cdots \\
& =\frac{1}{|\gamma+\lambda|}\left|y_{0}\right|+\frac{1}{|\gamma+\lambda|}\|\psi\|\|E\|\left\|y_{1}\right\|_{L^{1}[0, \infty)}+\frac{1}{|\gamma+\lambda|}\|\phi\|\left\|E_{0}\right\|\left\|z_{1}\right\|_{L^{1}[0, \infty)}
\end{aligned}
$$




$$
\begin{aligned}
& +\sum_{n=1}^{\infty} \lambda^{n-1}\|E\|^{n}\left\|y_{1}\right\|_{L^{1}[0, \infty)}+\sum_{n=1}^{\infty} \lambda^{n-1}\|E\|^{n}\left\|y_{2}\right\|_{L^{1}[0, \infty)} \\
& +\sum_{n=1}^{\infty} \lambda^{n-1}\|E\|^{n}\left\|y_{3}\right\|_{L^{1}[0, \infty)}+\sum_{n=1}^{\infty} \lambda^{n-1}\|E\|^{n}\left\|y_{4}\right\|_{L^{1}[0, \infty)} \\
& +\cdots \\
& +\sum_{n=1}^{\infty} \lambda^{n-1}\left\|E_{0}\right\|^{n}\left\|z_{1}\right\|_{L^{1}[0, \infty)}+\sum_{n=1}^{\infty} \lambda^{n-1}\left\|E_{0}\right\|^{n}\left\|z_{2}\right\|_{L^{1}[0, \infty)}+\sum_{n=1}^{\infty} \lambda^{n-1}\left\|E_{0}\right\|^{n}\left\|z_{3}\right\|_{L^{1}[0, \infty)} \\
& +\cdots \\
& =\frac{1}{|\gamma+\lambda|}\left|y_{0}\right|+\frac{1}{|\gamma+\lambda|}\|\psi\|\|E\|\left\|y_{1}\right\|_{L^{1}[0, \infty)}+\frac{1}{|\gamma+\lambda|}\|\phi\|\|\| E_{0}\|\| z_{1} \|_{L^{1}[0, \infty)} \\
& +\sum_{n=1}^{\infty} \lambda^{n-1}\|E\|^{n} \sum_{n=1}^{\infty}\left\|y_{n}\right\|_{L^{1}[0, \infty)}+\sum_{n=1}^{\infty} \lambda^{n-1}\left\|E_{0}\right\|^{n} \sum_{n=1}^{\infty}\left\|z_{n}\right\|_{L^{1}[0, \infty)} \\
& \leq \frac{1}{|\gamma+\lambda|}\left|y_{0}\right|+\frac{1}{|\gamma+\lambda|} \frac{\sup _{x \in[0, \infty)} b(x)}{\operatorname{Re} \gamma+\lambda+\inf _{x \in[0, \infty)} b(x)}\left\|y_{1}\right\|_{L^{1}[0, \infty)} \\
& +\frac{1}{|\gamma+\lambda|} \frac{\sup _{x \in[0, \infty)} b_{0}(x)}{\operatorname{Re} \gamma+\lambda+\inf _{x \in[0, \infty)} b_{0}(x)}\left\|z_{1}\right\|_{L^{1}[0, \infty)} \\
& +\frac{1}{\operatorname{Re} \gamma+\inf _{x \in[0, \infty)} b(x)} \sum_{n=1}^{\infty}\left\|y_{n}\right\|_{L^{1}[0, \infty)}+\frac{1}{\operatorname{Re} \gamma+\inf _{x \in[0, \infty)} b_{0}(x)} \sum_{n=1}^{\infty}\left\|z_{n}\right\|_{L^{1}[0, \infty)} \\
& =\sup \left\{\frac{1}{|\gamma+\lambda|}, \frac{1}{|\gamma+\lambda|} \frac{\sup _{x \in[0, \infty)} b(x)}{\operatorname{Re} \gamma+\lambda+\inf _{x \in[0, \infty)} b(x)}+\frac{1}{\operatorname{Re} \gamma+\inf _{x \in[0, \infty)} b(x)},\right. \\
& \left.\frac{1}{|\gamma+\lambda|} \frac{\sup _{x \in[0, \infty)} b_{0}(x)}{\operatorname{Re} \gamma+\lambda+\inf _{x \in[0, \infty)} b_{0}(x)}+\frac{1}{\operatorname{Re} \gamma+\inf _{x \in[0, \infty)} b_{0}(x)}\right\}\|(y, z)\|,
\end{aligned}
$$

which means that the result of this lemma is right.

Lemma 2.3 For $\gamma \in \rho\left(A_{0}\right)$ we have

$$
\begin{aligned}
& (p, Q) \in \operatorname{ker}\left(\gamma I-A_{m}\right) \quad \Longleftrightarrow \\
& p_{0}=\frac{1}{\gamma+\lambda}\left[b_{1} \int_{0}^{\infty} b_{0}(x) e^{-(\gamma+\lambda) x-\int_{0}^{x} b_{0}(\xi) \mathrm{d} \xi} \mathrm{d} x+a_{1} \int_{0}^{\infty} b(x) e^{-(\gamma+\lambda) x-\int_{0}^{x} b(\xi) \mathrm{d} \xi} \mathrm{d} x\right] \\
& p_{n}(x)=e^{-(\gamma+\lambda) x-\int_{0}^{x} b(\xi) \mathrm{d} \xi} \sum_{k=0}^{n-1} \frac{(\lambda x)^{k}}{k !} a_{n-k}, \quad n \geq 1, \\
& Q_{n}(x)=e^{-(\gamma+\lambda) x-\int_{0}^{x} b_{0}(\xi) \mathrm{d} \xi} \sum_{k=0}^{n-1} \frac{(\lambda x)^{k}}{k !} b_{n-k}, \quad n \geq 1, \\
& \left(a_{1}, a_{2}, \ldots\right) \in l^{1}, \quad\left(b_{1}, b_{2}, \ldots\right) \in l^{1} .
\end{aligned}
$$

Proof If $(p, Q) \in \operatorname{ker}\left(\gamma I-A_{m}\right)$, then $\left(\gamma I-A_{m}\right)(p, Q)=0$, which is equivalent to

$$
\begin{aligned}
& (\gamma+\lambda) p_{0}=\int_{0}^{\infty} Q_{1}(x) b_{0}(x) \mathrm{d} x+\int_{0}^{\infty} p_{1}(x) b(x) \mathrm{d} x, \\
& \frac{\mathrm{d} p_{1}(x)}{\mathrm{d} x}=-(\gamma+\lambda+b(x)) p_{1}(x),
\end{aligned}
$$




$$
\begin{aligned}
& \frac{\mathrm{d} p_{n}(x)}{\mathrm{d} x}=-(\gamma+\lambda+b(x)) p_{n}(x)+\lambda p_{n-1}(x), \quad \forall n \geq 2, \\
& \frac{\mathrm{d} Q_{1}(x)}{\mathrm{d} x}=-\left(\gamma+\lambda+b_{0}(x)\right) Q_{1}(x), \\
& \frac{\mathrm{d} Q_{n}(x)}{\mathrm{d} x}=-\left(\gamma+\lambda+b_{0}(x)\right) Q_{n}(x)+\lambda Q_{n-1}(x), \quad \forall n \geq 2 .
\end{aligned}
$$

By solving (2.37)-(2.40) we have

$$
\begin{aligned}
p_{1}(x)= & a_{1} e^{-(\gamma+\lambda) x-\int_{0}^{x} b(\xi) \mathrm{d} \xi}, \\
p_{n}(x)= & a_{n} e^{-(\gamma+\lambda) x-\int_{0}^{x} b(\xi) \mathrm{d} \xi} \\
& +\lambda e^{-(\gamma+\lambda) x-\int_{0}^{x} b(\xi) \mathrm{d} \xi} \int_{0}^{x} p_{n-1}(s) e^{(\gamma+\lambda) s+\int_{0}^{s} b(\xi) \mathrm{d} \xi} \mathrm{d} s, \quad n \geq 2, \\
Q_{1}(x)= & b_{1} e^{-(\gamma+\lambda) x-\int_{0}^{x} b_{0}(\xi) \mathrm{d} \xi}, \\
Q_{n}(x)= & b_{n} e^{-(\gamma+\lambda) x-\int_{0}^{x} b_{0}(\xi) \mathrm{d} \xi} \\
& +\lambda e^{-(\gamma+\lambda) x-\int_{0}^{x} b_{0}(\xi) \mathrm{d} \xi} \int_{0}^{x} Q_{n-1}(s) e^{(\gamma+\lambda) s+\int_{0}^{s} b_{0}(\xi) \mathrm{d} \xi} \mathrm{d} s, \quad n \geq 2 .
\end{aligned}
$$

Through inserting (2.41) and (2.43) into (2.36), it follows that

$$
\begin{aligned}
p_{0}= & \frac{a_{1}}{\gamma+\lambda} \int_{0}^{\infty} b(x) e^{-(\gamma+\lambda) x-\int_{0}^{x} b(\xi) \mathrm{d} \xi} \mathrm{d} x \\
& +\frac{b_{1}}{\gamma+\lambda} \int_{0}^{\infty} b_{0}(x) e^{-(\gamma+\lambda) x-\int_{0}^{x} b_{0}(\xi) \mathrm{d} \xi} \mathrm{d} x .
\end{aligned}
$$

By using (2.41), (2.42), (2.43) and (2.44) repeatedly, we deduce

$$
\begin{aligned}
& p_{2}(x)=a_{2} e^{-(\gamma+\lambda) x-\int_{0}^{x} b(\xi) d \xi}+\lambda e^{-(\gamma+\lambda) x-\int_{0}^{x} b(\xi) d \xi} \int_{0}^{x} a_{1} d s \\
& =e^{-(\gamma+\lambda) x-\int_{0}^{x} b(\xi) d \xi}\left[a_{2}+\lambda x a_{1}\right] \\
& p_{3}(x)=a_{3} e^{-(\gamma+\lambda) x-\int_{0}^{x} b(\xi) d \xi}+\lambda e^{-(\gamma+\lambda) x-\int_{0}^{x} b(\xi) d \xi} \int_{0}^{x}\left[a_{2}+\lambda s a_{1}\right] d s \\
& =e^{-(\gamma+\lambda) x-\int_{0}^{x} b(\xi) d \xi}\left[a_{3}+\lambda x a_{2}+\frac{(\lambda x)^{2}}{2} a_{1}\right], \\
& p_{4}(x)=a_{4} e^{-(\gamma+\lambda) x-\int_{0}^{x} b(\xi) d \xi}+\lambda e^{-(\gamma+\lambda) x-\int_{0}^{x} b(\xi) d \xi} \\
& \times \int_{0}^{x}\left[a_{3}+\lambda s a_{2}+\frac{(\lambda s)^{2}}{2} a_{1}\right] d s \\
& =e^{-(\gamma+\lambda) x-\int_{0}^{x} b(\xi) d \xi}\left[a_{4}+\lambda x a_{3}+\frac{(\lambda x)^{2}}{2} a_{2}+\frac{(\lambda x)^{3}}{3 !} a_{1}\right] \text {, } \\
& p_{n}(x)=e^{-(\gamma+\lambda) x-\int_{0}^{x} b(\xi) d \xi} \sum_{k=0}^{n-1} \frac{(\lambda x)^{k}}{k !} a_{n-k}, \quad n \geq 1, \\
& Q_{2}(x)=b_{2} e^{-(\gamma+\lambda) x-\int_{0}^{x} b_{0}(\xi) d \xi}+\lambda e^{-(\gamma+\lambda) x-\int_{0}^{x} b_{0}(\xi) d \xi} \int_{0}^{x} b_{1} d s \\
& =e^{-(\gamma+\lambda) x-\int_{0}^{x} b_{0}(\xi) d \xi}\left[b_{2}+\lambda x b_{1}\right],
\end{aligned}
$$




$$
Q_{n}(x)=e^{-(\gamma+\lambda) x-\int_{0}^{x} b_{0}(\xi) d \xi} \sum_{k=0}^{n-1} \frac{(\lambda x)^{k}}{k !} b_{n-k}, \quad n \geq 1 .
$$

Since $(p, Q) \in \operatorname{ker}\left(\gamma I-A_{m}\right)$, by the imbedding theorem in Adams [15],

$$
\begin{aligned}
\sum_{n=1}^{\infty}\left|a_{n}\right| & =\sum_{n=1}^{\infty}\left|p_{n}(0)\right| \leq \sum_{n=1}^{\infty}\left\|p_{n}\right\|_{L^{\infty}[0, \infty)} \\
& \leq \sum_{n=1}^{\infty}\left\{\left\|p_{n}\right\|_{L^{1}[0, \infty)}+\left\|\frac{d p_{n}}{d x}\right\|_{L^{1}[0, \infty)}\right\} \\
& <\infty \\
\sum_{n=1}^{\infty}\left|b_{n}\right| & =\sum_{n=1}^{\infty}\left|Q_{n}(0)\right| \leq \sum_{n=1}^{\infty}\left\|Q_{n}\right\|_{L^{\infty}[0, \infty)} \\
& \leq \sum_{n=1}^{\infty}\left\{\left\|Q_{n}\right\|_{L^{1}[0, \infty)}+\left\|\frac{d Q_{n}}{d x}\right\|_{L^{1}[0, \infty)}\right\} \\
& <\infty
\end{aligned}
$$

(2.45)-(2.54) show that (2.32)-(2.35) are true.

Conversely, if (2.32)-(2.35) hold, then by using the formulas

$$
\begin{aligned}
& \int_{0}^{\infty} e^{-C x} x^{k} \mathrm{~d} x=\frac{k !}{C^{k+1}}, \quad C>0, k \in \mathbb{N} \\
& \int_{0}^{\infty} b(x) e^{-\int_{0}^{x} b(\xi) d \xi} d x=1, \quad \int_{0}^{\infty} b_{0}(x) e^{-\int_{0}^{x} b_{0}(\xi) d \xi} d x=1,
\end{aligned}
$$

integration by parts and the Fubini theorem, we estimate

$$
\begin{aligned}
\left\|p_{n}\right\|_{L^{1}[0, \infty)} & =\int_{0}^{\infty}\left|e^{-(\gamma+\lambda) x-\int_{0}^{x} b(\xi) \mathrm{d} \xi} \sum_{k=0}^{n-1} \frac{(\lambda x)^{k}}{k !} a_{n-k}\right| \mathrm{d} x \\
& \leq \int_{0}^{\infty} e^{-(\operatorname{Re} \gamma+\lambda) x-\int_{0}^{x} b(\xi) \mathrm{d} \xi} \sum_{k=0}^{n-1} \frac{(\lambda x)^{k}}{k !}\left|a_{n-k}\right| \mathrm{d} x \\
& \leq \sum_{k=0}^{n-1} \frac{\lambda^{k}}{k !}\left|a_{n-k}\right| \int_{0}^{\infty} e^{-\left(\operatorname{Re} \gamma+\lambda+\inf _{x \in[0, \infty)} b(x)\right) x} x^{k} \mathrm{~d} x \\
& =\sum_{k=0}^{n-1} \frac{\lambda^{k}}{k !}\left|a_{n-k}\right| \frac{k !}{\left(\operatorname{Re} \gamma+\lambda+\inf _{x \in[0, \infty)} b(x)\right)^{k+1}} \\
& =\sum_{k=0}^{n-1} \frac{\lambda^{k}}{\left(\operatorname{Re} \gamma+\lambda+\inf _{x \in[0, \infty)} b(x)\right)^{k+1}}\left|a_{n-k}\right|, \quad n \geq 1
\end{aligned}
$$




$$
\begin{aligned}
& \left|p_{0}\right|+\sum_{n=1}^{\infty}\left\|p_{n}\right\|_{L^{1}[0, \infty)} \\
& \leq \frac{\left|a_{1}\right|}{|\gamma+\lambda|} \int_{0}^{\infty} b(x) e^{-\int_{0}^{x} b(\xi) \mathrm{d} \xi} \mathrm{d} x \\
& +\frac{\left|b_{1}\right|}{|\gamma+\lambda|} \int_{0}^{\infty} b_{0}(x) e^{-\int_{0}^{x} b_{0}(\xi) \mathrm{d} \xi} \mathrm{d} x \\
& +\sum_{n=1}^{\infty} \sum_{k=0}^{n-1} \frac{1}{\operatorname{Re} \gamma+\lambda+\inf _{x \in[0, \infty)} b(x)}\left(\frac{\lambda}{\operatorname{Re} \gamma+\lambda+\inf _{x \in[0, \infty)} b(x)}\right)^{k}\left|a_{n-k}\right| \\
& =\frac{\left|a_{1}\right|+\left|b_{1}\right|}{|\gamma+\lambda|} \\
& +\frac{1}{\operatorname{Re} \gamma+\lambda+\inf _{x \in[0, \infty)} b(x)} \sum_{k=0}^{\infty}\left(\frac{\lambda}{\operatorname{Re} \gamma+\lambda+\inf _{x \in[0, \infty)} b(x)}\right)^{k} \sum_{n=k+1}^{\infty}\left|a_{n-k}\right| \\
& =\frac{\left|a_{1}\right|+\left|b_{1}\right|}{|\gamma+\lambda|}+\frac{1}{\operatorname{Re} \gamma+\inf _{x \in[0, \infty)} b(x)} \sum_{n=1}^{\infty}\left|a_{n}\right| \\
& <\infty \text {, } \\
& \left\|Q_{n}\right\|_{L^{1}[0, \infty)} \leq \sum_{k=0}^{n-1} \frac{\lambda^{k}}{\left(\operatorname{Re} \gamma+\lambda+\inf _{x \in[0, \infty)} b_{0}(x)\right)^{k+1}}\left|b_{n-k}\right|, \quad n \geq 1 \\
& \Longrightarrow \\
& \sum_{n=1}^{\infty}\left\|Q_{n}\right\|_{L^{1}[0, \infty)} \leq \frac{1}{\operatorname{Re} \gamma+\lambda+\inf _{x \in[0, \infty)} b_{0}(x)} \\
& \times \sum_{n=1}^{\infty} \sum_{k=0}^{n-1}\left(\frac{\lambda}{\operatorname{Re} \gamma+\lambda+\inf _{x \in[0, \infty)} b_{0}(x)}\right)^{k}\left|b_{n-k}\right| \\
& =\frac{1}{\operatorname{Re} \gamma+\inf _{x \in[0, \infty)} b_{0}(x)} \sum_{n=1}^{\infty}\left|b_{n}\right| \\
& <\infty \text {. }
\end{aligned}
$$

(2.33) and (2.34) give

$$
\begin{aligned}
\frac{d p_{n}(x)}{d x}= & -(\gamma+\lambda+b(x)) e^{-(\gamma+\lambda) x-\int_{0}^{x} b(\xi) d \xi} \sum_{k=0}^{n-1} \frac{(\lambda x)^{k}}{k !} a_{n-k} \\
& +e^{-(\gamma+\lambda) x-\int_{0}^{x} b(\xi) d \xi} \sum_{k=1}^{n-1} \frac{\lambda^{k} x^{k-1}}{(k-1) !} a_{n-k} \\
= & -(\gamma+\lambda+b(x)) p_{n}(x)+\lambda p_{n-1}(x), \quad n \geq 2, \\
\frac{d p_{1}(x)}{d x}= & -(\gamma+\lambda+b(x)) p_{1}(x), \\
\frac{d Q_{n}(x)}{d x}= & -\left(\gamma+\lambda+b_{0}(x)\right) e^{-(\gamma+\lambda) x-\int_{0}^{x} b_{0}(\xi) d \xi} \sum_{k=0}^{n-1} \frac{(\lambda x)^{k}}{k !} b_{n-k} \\
& +e^{-(\gamma+\lambda) x-\int_{0}^{x} b_{0}(\xi) d \xi} \sum_{k=1}^{n-1} \frac{\lambda^{k} x^{k-1}}{(k-1) !} b_{n-k}
\end{aligned}
$$




$$
\begin{aligned}
& =-\left(\gamma+\lambda+b_{0}(x)\right) Q_{n}(x)+\lambda Q_{n-1}(x), \quad n \geq 2, \\
\frac{d Q_{1}(x)}{d x}= & -\left(\gamma+\lambda+b_{0}(x)\right) Q_{1}(x) .
\end{aligned}
$$

By combining (2.57), (2.58), (2.59) and (2.60) with (2.55) and (2.56), we derive

$$
\begin{aligned}
& \sum_{n=1}^{\infty}\left\|\frac{\mathrm{d} p_{n}(x)}{\mathrm{d} x}\right\|_{L^{1}[0, \infty)} \leq\left[\operatorname{Re} \gamma+2 \lambda+\sup _{x \in[0, \infty)} b(x)+|\operatorname{Im} \gamma|\right] \sum_{n=1}^{\infty}\left\|p_{n}\right\|_{L^{1}[0, \infty)} \\
& \quad<\infty \\
& \sum_{n=1}^{\infty}\left\|\frac{d Q_{n}}{d x}\right\|_{L^{1}[0, \infty)} \leq\left[\operatorname{Re} \gamma+2 \lambda+\sup _{x \in[0, \infty)} b_{0}(x)+|\operatorname{Im} \gamma|\right] \sum_{n=1}^{\infty}\left\|Q_{n}\right\|_{L^{1}[0, \infty)} \\
& \quad<\infty .
\end{aligned}
$$

(2.55)-(2.62) mean that $(p, Q) \in D\left(A_{m}\right)$ and $\left(\gamma I-A_{m}\right)(p, Q)=0$.

It is not difficult to see that $L$ is surjective. Moreover,

$$
\left.L\right|_{\operatorname{ker}\left(\gamma I-A_{m}\right)}: \operatorname{ker}\left(\gamma I-A_{m}\right) \rightarrow \partial X
$$

is invertible for $\gamma \in \rho\left(A_{0}\right)$. For $\forall \gamma \in \rho\left(A_{0}\right)$ we define the Dirichlet operator as

$$
D_{\gamma}:=\left(\left.L\right|_{\operatorname{ker}\left(\gamma I-A_{m}\right)}\right)^{-1}: \partial X \rightarrow \operatorname{ker}\left(\gamma I-A_{m}\right) .
$$

Lemma 2.3 gives the explicit form of $D_{\gamma}$ for $\gamma \in \rho\left(A_{0}\right)$

$$
\begin{aligned}
D_{\gamma}(\vec{a}, \vec{b})= & \left(\left(\begin{array}{ccccc}
\frac{1}{\gamma+\lambda} \psi \epsilon_{0} & 0 & 0 & \ldots \\
\epsilon_{0} & 0 & 0 & \ldots \\
\epsilon_{1} & \epsilon_{0} & 0 & \ldots \\
\epsilon_{2} & \epsilon_{1} & \epsilon_{0} & \ldots \\
\vdots & \vdots & \vdots & \ddots
\end{array}\right)\left(\begin{array}{c}
a_{1} \\
a_{2} \\
a_{3} \\
a_{4} \\
\vdots
\end{array}\right)+\left(\begin{array}{cccc}
\frac{1}{\gamma+\lambda} \phi \delta_{0} & 0 & 0 & \ldots \\
0 & 0 & 0 & \ldots \\
0 & 0 & 0 & \ldots \\
0 & 0 & 0 & \ldots \\
\vdots & \vdots & \vdots & \ddots
\end{array}\right)\left(\begin{array}{c}
b_{1} \\
b_{2} \\
b_{3} \\
b_{4} \\
\vdots
\end{array}\right)\right. \\
& \left.\left(\begin{array}{ccccc}
\delta_{0} & 0 & 0 & 0 & \ldots \\
\delta_{1} & \delta_{0} & 0 & 0 & \ldots \\
\delta_{2} & \delta_{1} & \delta_{0} & 0 & \ldots \\
\delta_{3} & \delta_{2} & \delta_{1} & \delta_{0} & \ldots \\
\vdots & \vdots & \vdots & \vdots & \ddots
\end{array}\right)\left(\begin{array}{c}
b_{1} \\
b_{2} \\
b_{3} \\
b_{4} \\
\vdots
\end{array}\right)\right)
\end{aligned}
$$

where

$$
\begin{array}{ll}
\epsilon_{k}=\frac{(\lambda x)^{k}}{k !} e^{-(\gamma+\lambda) x-\int_{0}^{x} b(\xi) \mathrm{d} \xi}, & k \geq 0, \\
\delta_{k}=\frac{(\lambda x)^{k}}{k !} e^{-(\gamma+\lambda) x-\int_{0}^{x} b_{0}(\xi) \mathrm{d} \xi}, & k \geq 0 .
\end{array}
$$


From (2.63) and the definition of $\Phi$, it is easy to determine the expression of $\Phi D_{\gamma}$ for $\gamma \in \rho\left(A_{0}\right)$.

$$
\begin{aligned}
\Phi D_{\gamma}(\vec{a}, \vec{b})= & \left(\begin{array}{ccccc}
\psi \epsilon_{1} & \psi \epsilon_{0} & 0 & 0 & \ldots \\
\psi \epsilon_{2} & \psi \epsilon_{1} & \psi \epsilon_{0} & 0 & \ldots \\
\psi \epsilon_{3} & \psi \epsilon_{2} & \psi \epsilon_{1} & \psi \epsilon_{0} & \ldots \\
\vdots & \vdots & \vdots & \vdots & \ddots
\end{array}\right)\left(\begin{array}{c}
a_{1} \\
a_{2} \\
a_{3} \\
\vdots
\end{array}\right) \\
& +\left(\begin{array}{ccccc}
\phi \delta_{1} & \phi \delta_{0} & 0 & 0 & \ldots \\
\phi \delta_{2} & \phi \delta_{1} & \phi \delta_{0} & 0 & \ldots \\
\phi \delta_{3} & \phi \delta_{2} & \phi \delta_{1} & \phi \delta_{0} & \ldots \\
\vdots & \vdots & \vdots & \vdots & \ddots
\end{array}\right)\left(\begin{array}{c}
b_{1} \\
b_{2} \\
b_{3} \\
\vdots
\end{array}\right), \\
& \left.\left(\begin{array}{cccc}
\frac{\lambda}{\gamma+\lambda} \psi \epsilon_{0} & 0 & 0 & \ldots \\
0 & 0 & 0 & \ldots \\
0 & 0 & 0 & \ldots \\
\vdots & \vdots & \vdots & \ddots
\end{array}\right)\left(\begin{array}{c}
a_{1} \\
a_{2} \\
a_{3} \\
\vdots
\end{array}\right)+\left(\begin{array}{cccc}
\frac{\lambda}{\gamma+\lambda} \phi \delta_{0} & 0 & 0 & \ldots \\
0 & 0 & 0 & \ldots \\
0 & 0 & 0 & \ldots \\
\vdots & \vdots & \vdots & \ddots
\end{array}\right)\left(\begin{array}{c}
b_{1} \\
b_{2} \\
b_{3} \\
\vdots
\end{array}\right)\right) .
\end{aligned}
$$

Haji and Radl [12] gave the following result through which we deduce the resolvent set of $A$ on the imaginary axis.

Lemma 2.4 If $\gamma \in \rho\left(A_{0}\right)$ and $1 \notin \sigma\left(\Phi D_{\gamma}\right)$, then

$$
\gamma \in \sigma(A) \Longleftrightarrow 1 \in \sigma\left(\Phi D_{\gamma}\right) \text {. }
$$

By using Lemma 2.4 and Nagel [14], page 297, we derive the following result.

Lemma 2.5 Let $b(x), b_{0}(x):[0, \infty) \rightarrow[0, \infty)$ be measurable, $0<\inf _{x \in[0, \infty)} b(x) \leq$ $\sup _{x \in[0, \infty)} b(x)<\infty$ and $0<\inf _{x \in[0, \infty)} b_{0}(x) \leq \sup _{x \in[0, \infty)} b_{0}(x)<\infty$. Then all points on the imaginary axis except zero belong to the resolvent set of $A$.

Proof Take $\gamma=i m, m \in \mathbb{R} \backslash\{0\}, \vec{a}=\left(a_{1}, a_{2}, \ldots\right) \in l^{1}$ and $\vec{b}=\left(b_{1}, b_{2}, \ldots\right) \in l^{1}$. Then by the Riemann-Lebesgue lemma,

$$
\begin{aligned}
& \lim _{m \rightarrow \infty} \int_{0}^{\infty} f(x) \cos (m x) d x=0, \\
& \lim _{m \rightarrow \infty} \int_{0}^{\infty} f(x) \sin (m x) d x=0, \quad f \in L^{1}[0, \infty), f(x) \geq 0,
\end{aligned}
$$

we know there exists $\mathcal{M}>0$ such that $|m|>\mathcal{M}$

$$
\begin{aligned}
\left|\int_{0}^{\infty} f(x) e^{-i m x} d x\right|^{2} & =\left(\int_{0}^{\infty} f(x) \cos (m x) d x\right)^{2}+\left(\int_{0}^{\infty} f(x) \sin (m x) d x\right)^{2} \\
& <\left(\int_{0}^{\infty} f(x) d x\right)^{2} \\
& \Longrightarrow \\
\left|\int_{0}^{\infty} f(x) e^{-i m x} d x\right| & <\int_{0}^{\infty} f(x) d x
\end{aligned}
$$


By replacing $f(x)$ in (2.64) with $f(x)=e^{-\lambda x-\int_{0}^{x} b(\xi) d \xi}, f(x)=e^{-\lambda x-\int_{0}^{x} b_{0}(\xi) d \xi}$ and using the fact

$$
\begin{aligned}
& \left|\int_{0}^{\infty} \frac{(\lambda x)^{n}}{n !} e^{-(i m+\lambda) x-\int_{0}^{x} b(\xi) d \xi} d x\right| \leq \int_{0}^{\infty} \frac{(\lambda x)^{n}}{n !} e^{-\lambda x-\int_{0}^{x} b(\xi) d \xi} d x, \quad n \geq 1, \\
& \left|\int_{0}^{\infty} \frac{(\lambda x)^{n}}{n !} e^{-(i m+\lambda) x-\int_{0}^{x} b_{0}(\xi) d \xi} d x\right| \leq \int_{0}^{\infty} \frac{(\lambda x)^{n}}{n !} e^{-\lambda x-\int_{0}^{x} b_{0}(\xi) d \xi} d x, \quad n \geq 1
\end{aligned}
$$

we derive, for $|m|>\mathcal{M}$,

$$
\begin{aligned}
& \left\|\Phi D_{\gamma}(\vec{a}, \vec{b})\right\|=\left|\psi \epsilon_{1} a_{1}+\psi \epsilon_{0} a_{2}+\phi \delta_{1} b_{1}+\phi \delta_{0} b_{2}\right| \\
& +\left|\psi \epsilon_{2} a_{1}+\psi \epsilon_{1} a_{2}+\psi \epsilon_{0} a_{3}+\phi \delta_{2} b_{1}+\phi \delta_{1} b_{2}+\phi \delta_{0} b_{3}\right| \\
& +\mid \psi \epsilon_{3} a_{1}+\psi \epsilon_{2} a_{2}+\psi \epsilon_{1} a_{3}+\psi \epsilon_{0} a_{4} \\
& +\phi \delta_{3} b_{1}+\phi \delta_{2} b_{2}+\phi \delta_{1} b_{3}+\phi \delta_{0} b_{4} \\
& +\cdots \\
& +\left|\sum_{j=0}^{n} \psi \epsilon_{j} a_{n+1-j}+\sum_{j=0}^{n} \phi \delta_{j} b_{n+1-j}\right| \\
& +\cdots \\
& +\left|\frac{\lambda}{\gamma+\lambda} \psi \epsilon_{0} a_{1}+\frac{\lambda}{\gamma+\lambda} \phi \delta_{0} b_{1}\right| \\
& \leq \sum_{n=1}^{\infty}\left|\psi \epsilon_{n}\right|\left|a_{1}\right|+\sum_{n=0}^{\infty}\left|\psi \epsilon_{n}\right|\left|a_{2}\right|+\sum_{n=0}^{\infty}\left|\psi \epsilon_{n}\right|\left|a_{3}\right|+\cdots \\
& +\sum_{n=1}^{\infty}\left|\phi \delta_{n}\right|\left|b_{1}\right|+\sum_{n=0}^{\infty}\left|\phi \delta_{n}\right|\left|b_{2}\right|+\sum_{n=0}^{\infty}\left|\phi \delta_{n}\right|\left|b_{3}\right|+\cdots \\
& +\frac{\lambda}{\sqrt{m^{2}+\lambda^{2}}}\left|\psi \epsilon_{0}\right|\left|a_{1}\right|+\frac{\lambda}{\sqrt{m^{2}+\lambda^{2}}}\left|\phi \delta_{0}\right|\left|b_{1}\right| \\
& <\sum_{n=1}^{\infty}\left|\psi \epsilon_{n}\right|\left|a_{1}\right|+\sum_{n=0}^{\infty}\left|\psi \epsilon_{n}\right| \sum_{k=2}^{\infty}\left|a_{k}\right| \\
& +\sum_{n=1}^{\infty}\left|\phi \delta_{n}\right|\left|b_{1}\right|+\sum_{n=0}^{\infty}\left|\phi \delta_{n}\right| \sum_{k=2}^{\infty}\left|b_{k}\right| \\
& +\left|\psi \epsilon_{0}\right|\left|a_{1}\right|+\left|\phi \delta_{0}\right|\left|b_{1}\right| \\
& =\sum_{n=0}^{\infty}\left|\psi \epsilon_{n}\right| \sum_{k=1}^{\infty}\left|a_{k}\right|+\sum_{n=0}^{\infty}\left|\phi \delta_{n}\right| \sum_{k=1}^{\infty}\left|b_{k}\right| \\
& =\sum_{n=0}^{\infty}\left|\int_{0}^{\infty} b(x) \frac{(\lambda x)^{n}}{n !} e^{-(i m+\lambda) x-\int_{0}^{x} b(\xi) d \xi} d x\right| \sum_{k=1}^{\infty}\left|a_{k}\right| \\
& +\sum_{n=0}^{\infty}\left|\int_{0}^{\infty} b_{0}(x) \frac{(\lambda x)^{n}}{n !} e^{-(i m+\lambda) x-\int_{0}^{x} b_{0}(\xi) d \xi} d x\right| \sum_{k=1}^{\infty}\left|b_{k}\right| \\
& <\sum_{n=0}^{\infty} \int_{0}^{\infty} b(x) \frac{(\lambda x)^{n}}{n !} e^{-\lambda x-\int_{0}^{x} b(\xi) d \xi} d x \sum_{k=1}^{\infty}\left|a_{k}\right|
\end{aligned}
$$




$$
\begin{aligned}
& +\sum_{n=0}^{\infty} \int_{0}^{\infty} b_{0}(x) \frac{(\lambda x)^{n}}{n !} e^{-\lambda x-\int_{0}^{x} b_{0}(\xi) d \xi} d x \sum_{k=1}^{\infty}\left|b_{k}\right| \\
& =\int_{0}^{\infty} b(x) \sum_{n=0}^{\infty} \frac{(\lambda x)^{n}}{n !} e^{-\lambda x-\int_{0}^{x} b(\xi) d \xi} d x \sum_{k=1}^{\infty}\left|a_{k}\right| \\
& +\int_{0}^{\infty} b_{0}(x) \sum_{n=0}^{\infty} \frac{(\lambda x)^{n}}{n !} e^{-\lambda x-\int_{0}^{x} b_{0}(\xi) d \xi} d x \sum_{k=1}^{\infty}\left|b_{k}\right| \\
& =\int_{0}^{\infty} b(x) e^{\lambda x} e^{-\lambda x-\int_{0}^{x} b(\xi) d \xi} d x \sum_{k=1}^{\infty}\left|a_{k}\right| \\
& +\int_{0}^{\infty} b_{0}(x) e^{\lambda x} e^{-\lambda x-\int_{0}^{x} b(\xi) d \xi} d x \sum_{k=1}^{\infty}\left|b_{k}\right| \\
& =\int_{0}^{\infty} b(x) e^{-\int_{0}^{x} b(\xi) d \xi} d x \sum_{k=1}^{\infty}\left|a_{k}\right| \\
& +\int_{0}^{\infty} b_{0}(x) e^{-\int_{0}^{x} b_{0}(\xi) d \xi} d x \sum_{k=1}^{\infty}\left|b_{k}\right| \\
& =-\left.e^{-\int_{0}^{x} b(\xi) d \xi}\right|_{0} ^{\infty} \sum_{k=1}^{\infty}\left|a_{k}\right|-\left.e^{-\int_{0}^{x} b_{0}(\xi) d \xi}\right|_{0} ^{\infty} \sum_{k=1}^{\infty}\left|b_{k}\right| \\
& =\sum_{k=1}^{\infty}\left|a_{1}\right|+\sum_{k=1}^{\infty}\left|b_{k}\right| \\
& =\|(\vec{a}, \vec{b})\| \\
& \Longrightarrow
\end{aligned}
$$

$\left\|\Phi D_{\gamma}\right\|<1$

(2.65) means that when $|m|>\mathcal{M}$, the spectral radius $r\left(\Phi D_{\gamma}\right) \leq\left\|\Phi D_{\gamma}\right\|<1$, which implies $1 \notin \sigma\left(\Phi D_{\gamma}\right)$ for $|m|>\mathcal{M}$, and therefore by Lemma 2.4 , we know $\gamma=i m \notin \sigma(A)$ for $|m|>$ $\mathcal{M}$, that is,

$$
\{i m|| m \mid>\mathcal{M}\} \subset \rho(A), \quad\{i m|| m \mid \leq \mathcal{M}\} \subset \sigma(A) \cap i \mathbb{R}
$$

On the other hand, since $T(t)$ is positive uniformly bounded by Theorem 1.1, by Corollary 2.3 in Nagel [14], page 297, we know that $\sigma(A) \cap i \mathbb{R}$ is imaginary additively cyclic, which states that $i m \in \sigma(A) \cap i \mathbb{R} \Rightarrow i m k \in \sigma(A) \cap i \mathbb{R}$ for all integer $k$, from which together with (2.66) and Lemma 2.1 we conclude $\sigma(A) \cap i \mathbb{R}=\{0\}$.

It is not difficult to prove $X^{*}$, dual space of $X$, is as follows:

$$
X^{*}=\left\{\begin{array}{l|l}
\left(p^{*}, Q^{*}\right) & \left.\begin{array}{l}
p^{*} \in \mathbb{R} \times L^{\infty}[0, \infty) \times L^{\infty}[0, \infty) \times \cdots, \\
Q^{*} \in L^{\infty}[0, \infty) \times L^{\infty}[0, \infty) \times L^{\infty}[0, \infty) \times \cdots, \\
\left\|\left(p^{*}, Q^{*}\right)\right\|=\max \left\{\begin{array}{l}
\sup \left\{\left|p_{0}^{*}\right|, \sup _{n \geq 1}\left\|p_{n}^{*}\right\|_{L^{\infty}[0, \infty)}\right\}, \\
\sup _{n \geq 1}\left\|Q_{n}^{*}\right\|_{L^{\infty}[0, \infty)}
\end{array}\right.
\end{array}\right\}<\infty
\end{array}\right\} .
$$


It is obvious that $X^{*}$ is a Banach space. Gupur [4] gave the expression of $A^{*}$, the adjoint operator of $A$ as follows:

$$
A^{*}\left(p^{*}, Q^{*}\right)=(G+F+\Re)\left(p^{*}, Q^{*}\right), \quad\left(p^{*}, Q^{*}\right) \in D(G),
$$

where

$$
\begin{aligned}
& G\left(p^{*}, Q^{*}\right)=\left(\begin{array}{ccccc}
-\lambda & 0 & 0 & 0 & \ldots \\
0 & \frac{d}{d x}-(\lambda+b(x)) & 0 & 0 & \ldots \\
0 & 0 & \frac{d}{d x}-(\lambda+b(x)) & 0 & \ldots \\
0 & 0 & 0 & \frac{d}{d x}-(\lambda+b(x)) & \ldots \\
\vdots & \vdots & \vdots & \vdots & \ddots
\end{array}\right)\left(\begin{array}{c}
p_{0}^{*} \\
p_{1}^{*}(x) \\
p_{2}^{*}(x) \\
p_{3}^{*}(x) \\
\vdots
\end{array}\right) \text {, } \\
& \left.\left(\begin{array}{cccc}
\frac{d}{d x}-\left(\lambda+b_{0}(x)\right) & 0 & 0 & \ldots \\
0 & \frac{d}{d x}-\left(\lambda+b_{0}(x)\right) & 0 & \ldots \\
0 & 0 & \frac{d}{d x}-\left(\lambda+b_{0}(x)\right) & \ldots \\
\vdots & \vdots & \vdots & \ddots
\end{array}\right)\left(\begin{array}{c}
Q_{1}^{*}(x) \\
Q_{2}^{*}(x) \\
Q_{3}^{*}(x) \\
\vdots
\end{array}\right)\right) \text {, } \\
& D(G)=\left\{\begin{array}{l|l}
\left(p^{*}, Q^{*}\right) \in X^{*} & \begin{array}{l}
\frac{d p_{k}^{*}(x)}{d x} \text { and } \frac{d Q_{k}^{*}(x)}{d x} \text { exist and } \\
p_{k}^{*}(\infty)=Q_{k}^{*}(\infty)=\alpha, k \geq 1
\end{array}
\end{array}\right\}, \\
& F\left(p^{*}, Q^{*}\right)=\left(\left(\begin{array}{cccc}
0 & 0 & 0 & \cdots \\
b(x) & 0 & 0 & \ldots \\
0 & b(x) & 0 & \ldots \\
0 & 0 & b(x) & \ldots \\
\vdots & \vdots & \vdots & \ddots
\end{array}\right)\left(\begin{array}{c}
p_{0}^{*} \\
p_{1}^{*}(0) \\
p_{2}^{*}(0) \\
p_{3}^{*}(0) \\
\vdots
\end{array}\right)\right. \\
& \left.\left(\begin{array}{ccccc}
b_{0}(x) & 0 & 0 & 0 & \ldots \\
0 & b_{0}(x) & 0 & 0 & \ldots \\
0 & 0 & b_{0}(x) & 0 & \ldots \\
0 & 0 & 0 & b_{0}(x) & \ldots \\
\vdots & \vdots & \vdots & \vdots & \ddots
\end{array}\right)\left(\begin{array}{c}
p_{0}^{*} \\
p_{1}^{*}(0) \\
p_{2}^{*}(0) \\
p_{3}^{*}(0) \\
\vdots
\end{array}\right)\right) \\
& \Re\left(p^{*}, Q^{*}\right)=\left(\begin{array}{cccc}
\lambda & 0 & 0 & \ldots \\
0 & 0 & 0 & \ldots \\
0 & 0 & 0 & \ldots \\
\vdots & \vdots & \vdots & \ddots
\end{array}\right)\left(\begin{array}{c}
Q_{1}^{\prime \prime}(0) \\
Q_{2}^{*}(0) \\
Q_{3}^{*}(0) \\
\vdots
\end{array}\right)+\left(\begin{array}{ccccc}
0 & 0 & 0 & 0 & \ldots \\
0 & 0 & \lambda & 0 & \ldots \\
0 & 0 & 0 & \lambda & \ldots \\
\vdots & \vdots & \vdots & \vdots & \ddots
\end{array}\right)\left(\begin{array}{c}
p_{0}^{*} \\
p_{1}^{*}(x) \\
p_{2}^{*}(x) \\
\vdots
\end{array}\right) \text {, } \\
& \left.\left(\begin{array}{ccccc}
0 & \lambda & 0 & 0 & \cdots \\
0 & 0 & \lambda & 0 & \cdots \\
0 & 0 & 0 & \lambda & \cdots \\
\vdots & \vdots & \vdots & \vdots & \ddots
\end{array}\right)\left(\begin{array}{c}
Q_{1}^{*}(x) \\
Q_{2}^{*}(x) \\
Q_{3}^{*}(x) \\
\vdots
\end{array}\right)\right) \text {. }
\end{aligned}
$$

Since $T(t)$ is uniformly bounded, by Arendt and Batty [16] and Lemma 2.1, we know that 0 is an eigenvalue of $A^{*}$. Furthermore, by replacing $\mu$ and $\eta$ in Lemma 3 in Gupur [4] with $b(x)$ and $b_{0}(x)$, respectively, we deduce the following result. 
Lemma 2.6 If $\int_{0}^{\infty} \lambda x b(x) e^{-\int_{0}^{x} b(\xi) d \xi} d x<1$, then 0 is an eigenvalue of $A^{*}$ with geometric multiplicity one.

Since Theorem 1.1, Lemma 2.1, Lemma 2.5 and Lemma 2.6 satisfy the conditions of Theorem 14 in Gupur, Li and Zhu [7], the following conclusion is the direct result of Theorem 14 in Gupur, Li and Zhu [7].

Theorem 2.7 Let $b(x), b_{0}(x):[0, \infty) \rightarrow[0, \infty)$ be measurable, $0<\inf _{x \in[0, \infty)} b(x) \leq$ $\sup _{x \in[0, \infty)} b(x)<\infty$ and $0<\inf _{x \in[0, \infty)} b_{0}(x) \leq \sup _{x \in[0, \infty)} b_{0}(x)<\infty$. If $\int_{0}^{\infty} \lambda x b(x) \times$ $e^{-\int_{0}^{x} b(\xi) d \xi} d x<1$, then the time-dependent solution of the system (1.9) converges strongly to its steady-state solution, that is,

$$
\lim _{t \rightarrow \infty}\left\|(p, Q)(\cdot, t)-\left\langle\left(p^{*}, Q^{*}\right),(p(0), Q(0))\right\rangle(p, Q)(\cdot)\right\|=0,
$$

where $\left(p^{*}, Q^{*}\right)$ and $(p, Q)$ are the eigenvectors in Lemma 2.6 and Lemma 2.1, respectively.

When $b(x)=\mu$ and $b_{0}(x)=\eta$, Lin and Gupur [9] proved that if $\sqrt{\lambda}<\sqrt{\mu}<\sqrt{\lambda}+\sqrt{\eta}$, then $(2 \sqrt{\lambda \mu}-\lambda-\mu) \theta$ are eigenvalues of $A$ with geometric multiplicity one for all $\theta \in(0,1)$. Which means that the result in Theorem 2.7 is optimal, that is to say, it is impossible that the time-dependent solution of the system (1.9) exponentially converges to its steady-state solution.

\section{Competing interests}

The authors declare that they have no competing interests.

Authors' contributions

All authors read and approved the final manuscript.

\section{Author details}

${ }^{1}$ College of Mathematics and Systems Science, Xinjiang University, Urumqi, 830046, P.R. China. ${ }^{2}$ School of Mathematical Sciences, Xinjiang Normal University, Urumqi, 830054, P.R. China.

\section{Acknowledgements}

This work was supported by the Natural Science Foundation of Xinjiang (No: 2012211A023).

Received: 6 October 2012 Accepted: 22 January 2013 Published: 11 February 2013

\section{References}

1. Takagi, H: Time-dependent analysis of M/G/1 vacation models with exhaustive service. Queueing Syst. 6, 369-390 (1990)

2. Welch, PD: On a generalized M/G/1 queueing process in which the first customer of each period receives exceptional service. Oper. Res. 12, 736-752 (1964)

3. Minh, DL: Transient solutions of some exhaustive-service M/G/1 queues with generalized independent vacations. Cent. Eur. J. Oper. Res. 36, 197-201 (1998)

4. Gupur, G: Asymptotic property of the solution of $M / M / 1$ queueing model with exceptional service time for the first customer in each busy period. Int. J. Differ. Equ. Appl. 8, 23-94 (2003)

5. Gupur, G: Time-dependent analysis for a queue modeled by an infinite system of partial differential equations. Sci. China Math. 55, 985-1004 (2012)

6. Gupur, G: Semigroup method for M/G/1 queueing system with exceptional service time for the first customer in each busy period. Indian J. Math. 44, 125-146 (2002)

7. Gupur, G, Li, XZ, Zhu, GT: Functional Analysis Method in Queueing Theory. Research Information Ltd, Herdfortshire (2001)

8. Zhang, MQ, Gupur, G: Another eigenvalue of the M/M/1 queueing model with exceptional service times for the first customer in each busy period. Acta Anal. Funct. Appl. 11, 62-68 (2009)

9. Lin, XJ, Gupur, G: Other eigenvalues of the M/M/1 queueing model with exceptional service times for the first customer in each busy period. Acta Anal. Funct. Appl. 13, 383-391 (2011)

10. Gupur, G: Asymptotic property of the solution of a repairable, standby, human and machine system. Int. J. Pure Appl. Math. 8, 35-54 (2006)

11. Greiner, G: Perturbing the boundary conditions of a generator. Houst. J. Math. 13, 213-229 (1987) 
12. Haji, A, Radl, A: Asymptotic stability of the solution of the $M / M^{B} / 1$ queueing model. Comput. Math. Appl. 53, 1411-1420 (2007)

13. Gupur, G: Advances in queueing models' research. Acta Anal. Funct. Appl. 13, 225-245 (2011)

14. Nagel, R (ed.): One-Parameter Semigroups of Positive Operators. Springer, Berlin (1986) LNM 1184

15. Adams, R: Sobolev Spaces. Academic Press, New York (1975)

16. Arendt, W, Batty, CJK: Tauberian theorems and stability of one-parameter semigroups. Trans. Am. Math. Soc. 13, 837-852 (1988)

doi:10.1186/1687-2770-2013-17

Cite this article as: Gupur and Ehmet: Asymptotic behavior of the time-dependent solution of an M/G/1 queueing model. Boundary Value Problems 2013 2013:17.

\section{Submit your manuscript to a SpringerOpen ${ }^{\circ}$} journal and benefit from:

- Convenient online submission

Rigorous peer review

- Immediate publication on acceptance

- Open access: articles freely available online

- High visibility within the field

- Retaining the copyright to your article 